\title{
CYCLE BIOLOGIQUE ET PRODUCTION DE CAPNIONEURA BRACHYPTERA D. (PLÊCOPTẼRES) DANS UN RUISSEAU D'ALTITUDE DES PYRENEES CENTRALES
}

\author{
par P. LAVANDiER ${ }^{1}$.
}

\begin{abstract}
Le cycle de développement de Capnioneura brachyptera dure deux ans dans un ruisseau des Pyrénées centrales, à 1950 mètres d'altitude. Le premier hiver est passé à l'état d'œuf ; la croissance, très faible la première année, devient très rapide au cours du deuxième été dès la fin de la fonte des neiges.

La production varie peu d'une cohorte à la suivante et correspond, pour $70 \%$, à la croissance de deuxième année. La mortalité larvaire paraît faible. Le rapport $\mathrm{P} / \mathrm{B}$ max., compris entre 1,5 et 2 est voisin de ceux déjà mentionnés pour d'autres espèces des Pyrénées centrales.
\end{abstract}

\section{Life cycle and production of Capnioneura brachyptera D. (Plecoptera)}

in a high mountain stream of the Central Pyrenees.

The life cycle of Capnioneura brachytera lasts two years in a Central Pyrenean stream at an altitude of $1950 \mathrm{~m}$. The first winter is spent in the egg stage; growth is very slow in the first year but becomes very rapid during the second summer from the time when the snow melting ends.

The production shows little variation from one cohort to the next. $70 \%$ of the production occurs during the growth peliod of the second year. Larval mortality is apparently low. The ratio $\mathrm{P} / \mathrm{B}$ max. lies between 1.5 and 1.2 , and is close to those values already found for other aquatic insects of the Central Pyrenees.

La prospection intensive du réseau hydrographique du vallon d'Estaragne dans les Pyrénées centrales a permis la récolte de plusieurs espèces endémiques pyrénéennes. Capnioneura brachyptera Despax figure parmi les formes les plus abondantes de cette faune dont nous abordons ici l'étude. C'est une espèce automnale de haute altitude, l'une des six espèces de Capniidae présentes dans les Pyrénées (Berthélémy 1966, 1969).

\section{1. - MILIEU ÉTUDIE - MATERIEL - METHODES}

Le ruisseau étudié correspond à l'affluent a 6 dans la description du réseau hydrographique de l'Estaragne (Lavandier 1974). Il présente une forte pente et un lit riche en mousses dans la pre-

1. Laboratoire d'Hydrobiologie, Université Paul-Sabatier, 118, route de Narbonne, 31077 Toulouse Cedex. 
mière partie de son cours. Il s'étale ensuite largement sur un replat avant de joindre le torrent à $1930 \mathrm{~m}$ d'altitude. La station se situe dans la partie initiale du replat. A ce niveau :

- le débit peut atteindre $200 \mathrm{l} / \mathrm{s}$ à la fonte des neiges ou après de gros orages, avec des vitesses de courant supérieures à $1 \mathrm{~m} / \mathrm{s}$; il est souvent inférieur à $20 \mathrm{l} / \mathrm{s}$ à l'éliage ;

- le fond, dépourvu de végétation est formé de galets; il est riche en matières organiques et débris végétaux arrachés à l'amont ;

-. la station est enneigée 4 à 5 mois par an (décembre - avril) ;

- les températures extrêmes sont de $0^{\circ} \mathrm{C}$ (novembre) et $8{ }^{\circ} \mathrm{C}$ (août).

Les valeurs instantanées relevées chaque semaine en milieu d'après-midi donnent une image des variations de la température à la station (fig. 1).
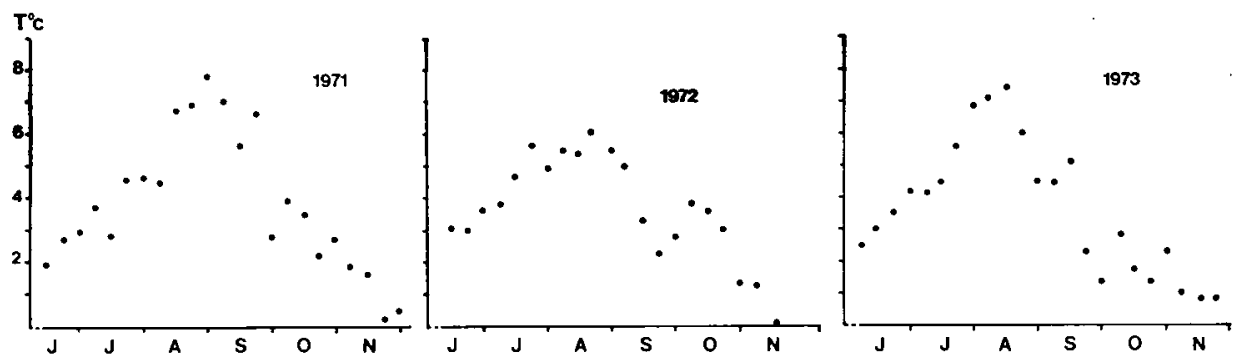

Fig. 1. - Température de l'eau à la station. Valeurs instantanées relevées chaque semaine en milieu d'après-midi.

La faune a été étudiée durant trois années consécutives pendant la période d'accessibilité du ruisseau.

'Les adultes ont été capturés par piégeage et chasse. Au cours des trois années d'observations nous avons effectué une demiheure de chasse hebdomadaire à l'aide d'un filet-fauchoir. En 1971 et 1972 un piège pyramidal à surface de capture triangulaire de $1 / 8 \mathrm{de}^{2}$ a permis de recueillir les imagos à leur émergence. Une vingtaine de femelles ont été disséquées afin d'apprécier l'état de maturation des ovaires.

La population larvaire a été suivie, à raison de deux prélèvements tous les quinze jours la première année, de deux prélèvements mensuels les années suivantes. Chaque relevé (un au milieu du ruisseau, l'autre près des rives) est effectué sur une surface de $0,1 \mathrm{~m}^{2}$ avec un filet du Surber (vide de maille de $0,15 \mathrm{~mm}$ ). Fixée au formol à $4 \%$ la faune est ensuite triée sous la loupe binoculaire.

Pour étudier la croissance larvaire, nous avons mesuré la largeur de la capsule céphalique (yeux compris) de toutes les larves 
recueillies, en séparant dès que possible mâles et femelles. En outre, il semble que la taille des fourreaux alaires permette de distinguer les cinq derniers stades larvaires, malgré les fortes variations individuelles que présente cette espèce brachyptère. La figure 2 donne à titre indicatif la relation existant entre la largeur de la capsule céphalique, la longueur des fourreaux alaires et la taille de l'animal (cerques non compris).

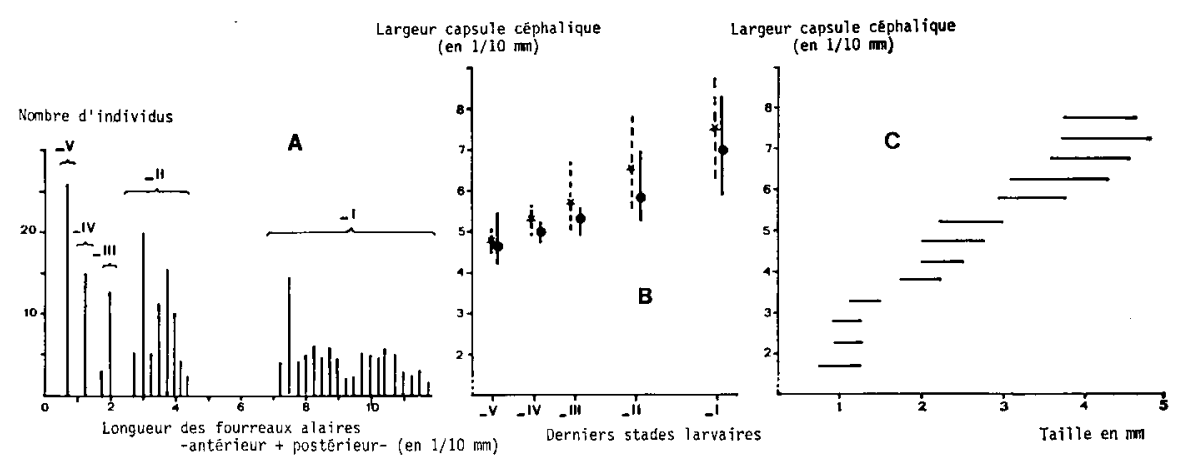

FIG. 2. - A : Distinction des derniers stades larvaires précédant la mue imaginale (Imago - 1 à Imago -5). B : Relation largeur de capsule céphalique - derniers stades larvaires. C : Relation largeur de capsule céphalique taille de l'animal (cerques non compris).

La biomasse est calculée à partir de larves formolécs issues des prélèvements. Différents lots de larves groupées par classe de largeur de capsule céphalique sont pesés après séchage de l'eau superficielle (poids frais) et après passage à l'étuve à $80^{\circ} \mathrm{C}$ durant $24 \mathrm{~h}$ (poids sec). Nombre d'individus et biomasse sont exprimés par $\mathbf{m}^{2}$ de ruisseau.

\section{2. - RẼSULTATS}

\section{1. - Cycle de développement.}

Les premiers adultes de Capnioneura brachyptera apparaissent dans la première quinzaine de septembre, les mâles étant plus précoces que les femelles; celles-ci dominent dans le piège à la fin de l'année ( $f i g .3$ ). Sur l'ensemble des adultes piégés, le sex-ratio est égal à 1 ; par contre les mâles dominent nettement dans les captures par chasse. Les adultes se nourrissent d'éléments végétaux. La dissection d'un certain nombre d'imagos montre que les femelles piégées ne sont pas prêtes à pondre immédiatement alors que celles capturées par chasse fin octobre étaient toutes mûres. Le nombre des œufs comptés dans l'abdomen des femelles variait de 70 à 280 (moyenne 155 pour 15 femelles). 


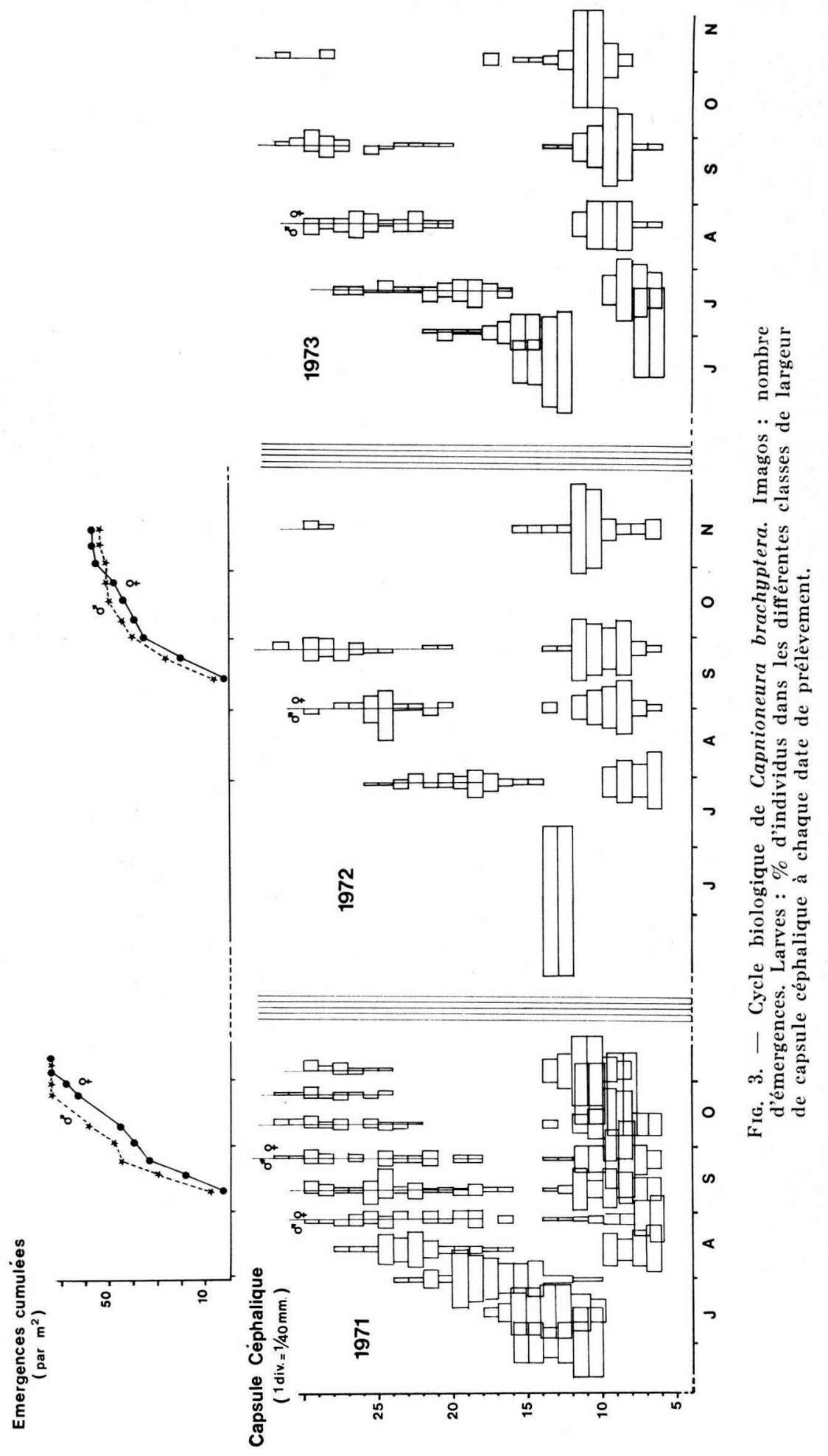


Les larvules ne sont récoltées qu'en juillet mais persistent dans les prélèvements jusqu'en septembre ; l'hiver et la période de fonte des neiges se passent donc à l'état d'œuf ou de très petites larves non capturées. Pendant le premier été, l'accroissement est très lent: le deuxième hiver survient alors que les larves n'ont pas encore d'ébauches de fourreaux alaires. L'été suivant, par contre, la croissance est extrêmement rapide et les adultes les plus précoces s'envolent dès le début septembre.

\section{2. - Croissance pondérale.}

La relation entre la largeur de la capsule céphalique et le poids moyen d'une larve (Poids Sec et Poids Frais) est représentée dans la figure 4. Les droites de régression obtenues permettent d'éva-

Largeur capsule cephalique

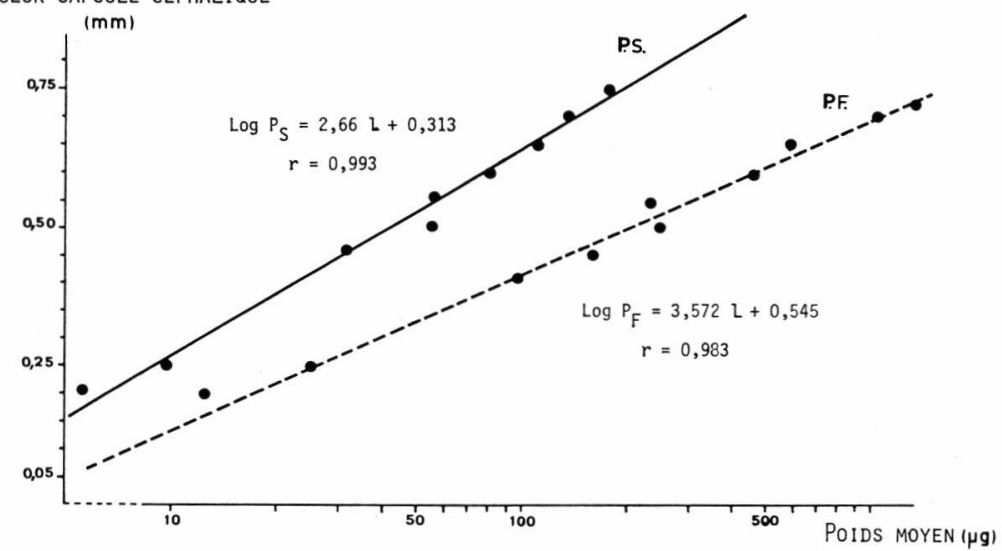

FIg. 4. - Relation largeur de capsule céphalique - poids sec (trait continu) et largeur de capsule céphalique - poids frais (tirets) chez Capnioneura brachyptera.

luer le poids moyen (PS et PF) de la larve au cours de son développement. La pente des deux droites est différente : le rapport $\mathrm{PS} / \mathrm{PF}$ diminue durant la croissance larvaire. Il passe de 0,38 chez les plus petites larves récoltées à 0,13 chez les plus grandes ; seule cette dernière valeur se rapproche du facteur de conversion $\mathrm{PS} / \mathrm{PF}=0,15$ généralement utilisé pour les invertébrés du benthos (Kajak 1967). Compte tenu de l'évolution de ce rapport au cours du développement et de la précision généralement plus grande des pesées de matières sèches, nous avons exprimé nos résultats de croissance, biomasse et production en poids sec.

La représentation graphique de la croissance pondérale de Capnioneura brachyptera (fig. 5) met en évidence les points suivants : 1) le développement présente deux phases distinctes caractérisées chacune par un taux de croissance propre ; 2 ) la croissance hiver- 


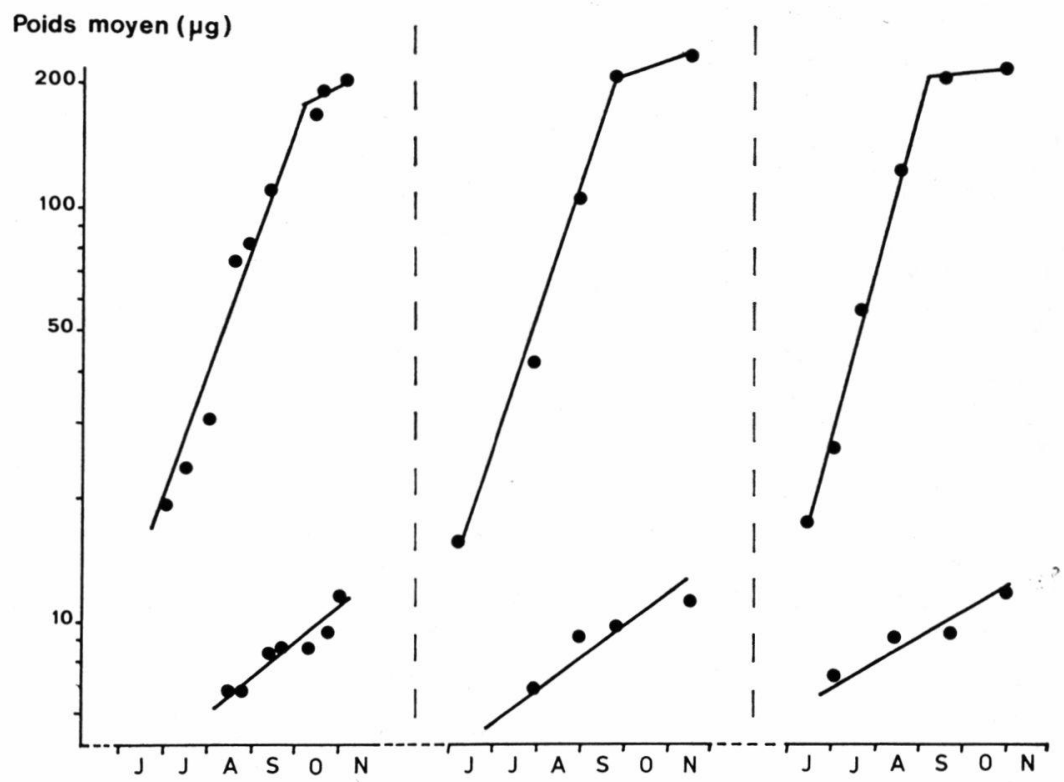

Fig. 5. - Croissance du poids moyen chez Capnioneura brachyptera en 1971, 1972 et 1973 .

nale est négligeable; 3 ) les cohortes successives ont des taux d'accroissement similaires. La légère augmentation du poids moyen notée au terme de la croissance et traduite par la cassure des courbes, est due à la prédominance des larves femelles sur les mâles à la fin du cycle biologique.

\section{3. - Evvolution des nombres et des biomasses.}

L'évolution des nombres représentée dans la figure 6 se traduit par un tracé en dent de scie dû aux fluctuations de l'échantillonnage. La distribution inégale de la faune est soulignée par la grande différence d'effectifs que présentent les deux relevés réalisés lors de chaque échantillonnage. Plusieurs phénomènes se dégagent néanmoins :

1) la mortalité paraît très faible : le nombre maximum de larves de l'année est à peine trois fois supérieur au nombre de larves âgées d'un an au début de la période de vol ;

2 ) lors de la fonte des neiges, on observe une très forte diminution du nombre de larves capturées. Cette diminution est sans doute liée à des déplacements de la faune. La densité larvaire redevient normale par la suite ;

3 ) ces migrations, jointes à l'étalement des éclosions déterminent une augmentation progressive du nombre de jeunes larves;

4) les plus fortes densités larvaires s'observent au début de la 

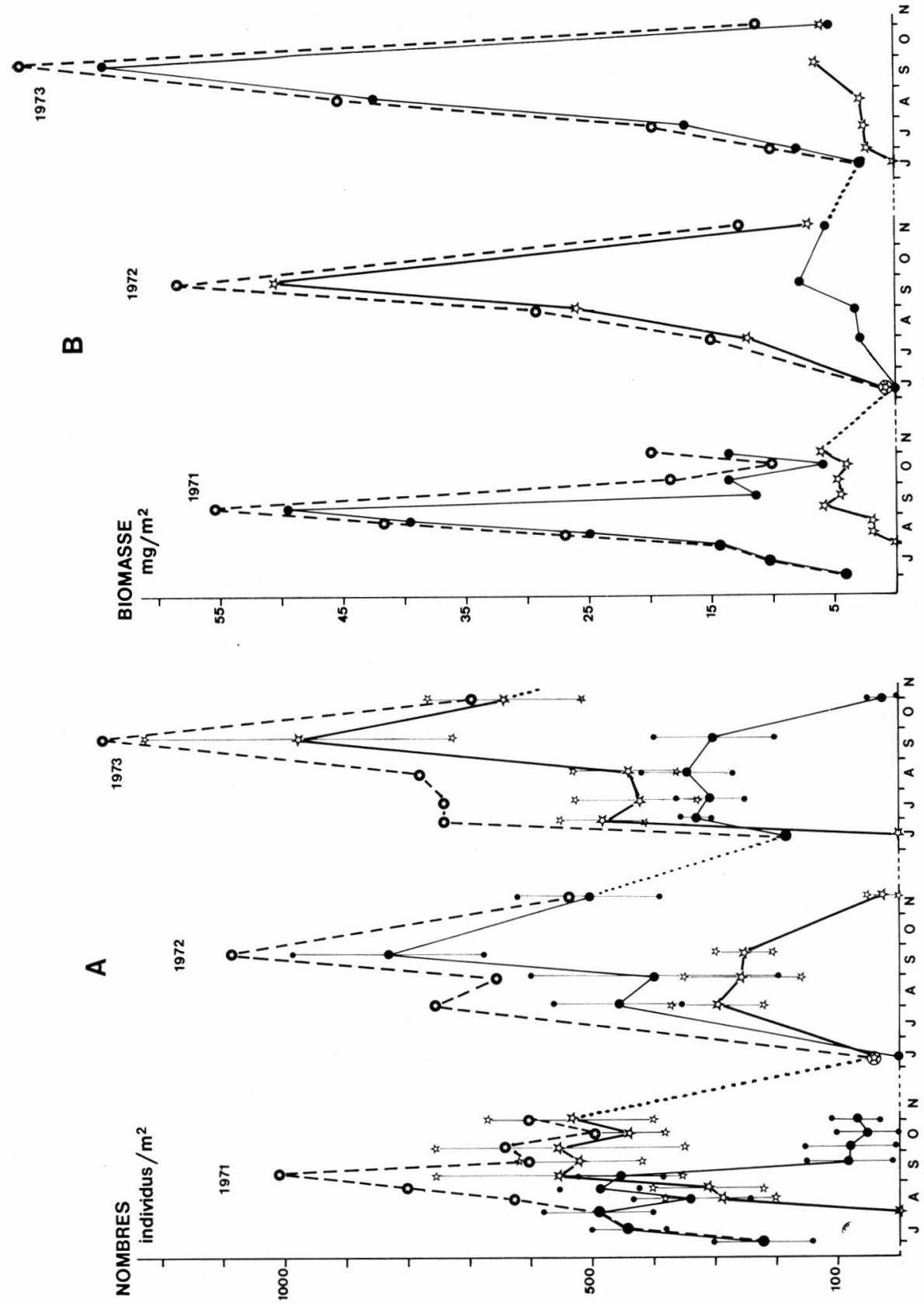

Fig. 6. - A) Traits continus : Évolution numérique des cohortes; les traits verticaux indiquent l'écart d'effectif noté entre les deux prélèvements qui constituent chaque échantillonnage. - Tirets : Évolution numérique de la population, les effectifs des deux générations étant cumulés.

B) Traits continus : Evolution de la biomasse moyenne de chaque cohorte. - Tirets : Evolution de la biomasse de la population, les biomasses des deux générations étant cumulées. 
période de vol, alors que la génération âgée est encore aquatique et que la nouvelle éclôt ;

$5)$ entre deux cohortes successives, l'évolution de la population présente de grandes analogies, que l'on considère les générations de première ou de deuxième année.

Étant donné les différences annuelles de croissance présentées par les larves au cours de leur développement et le maintien de l'effectif des cohortes au long du cycle biologique, l'importance et l'évolution des biomasses sont essentiellement déterminées par la population âgée. La biomasse maximale se rencontre au début de la période de vol.

\section{4. - Production.}

Nous avons estimé la production d'après la méthode graphique d'Allen (1951) à partir de courbe de croissance-survie établie pour

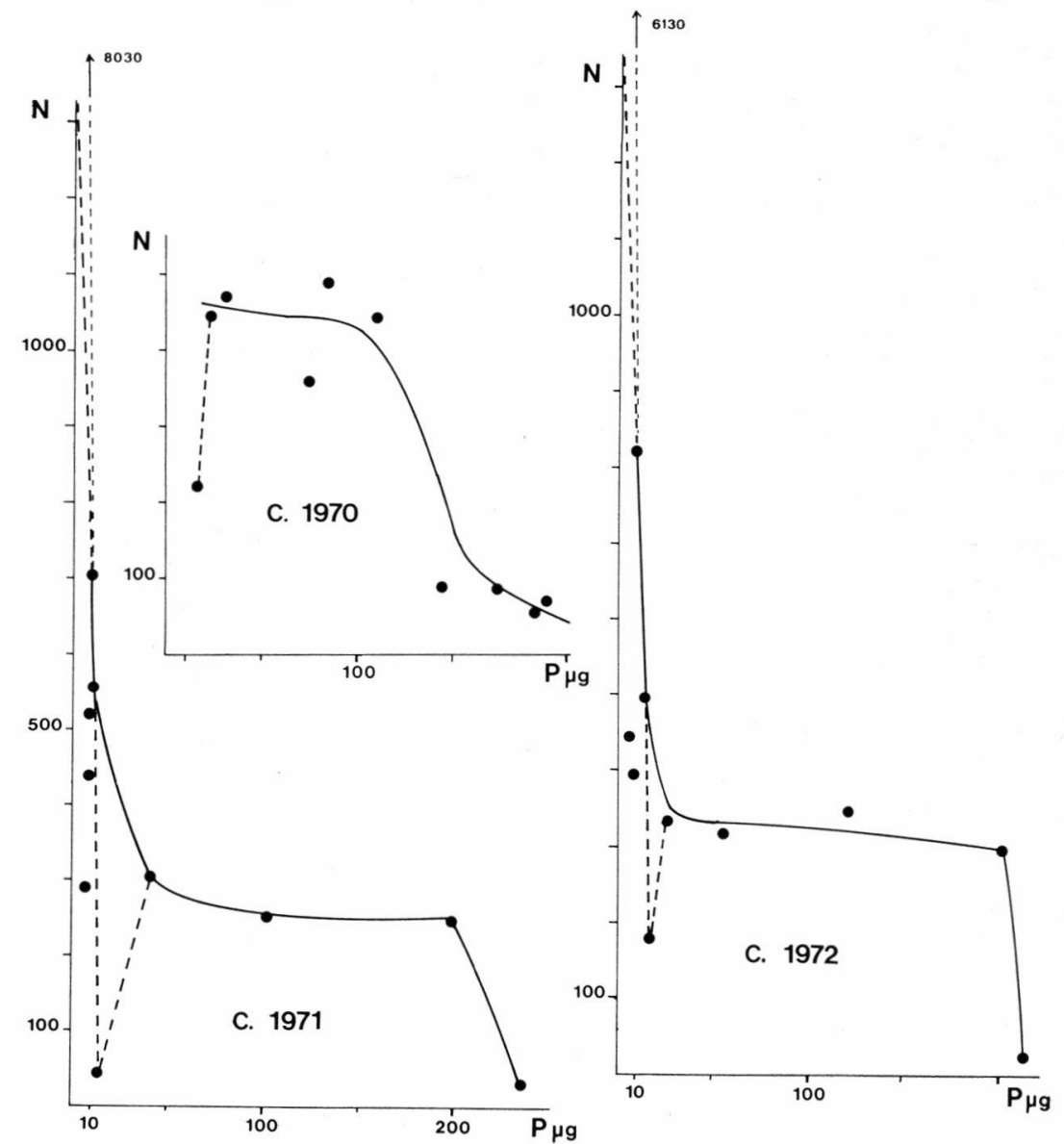

Fig. 7. - Courbe de croissance-survie de plusieurs cohortes de Capnioneura brachyptera. 
chaque cohorte. Malgré le tracé approché des courbes, cette méthode simple et rapide donne des résultats comparables à ceux obtenus par calcul (Elliott 1973, Décamps et Lafont 1974). L'observation des courbes (fig. 7) pose deux problèmes : la présence d'une forte discontinuité d'une part, l'estimation de l'ordonnée à l'origine d'autre part.

- La discontinuité traduit la diminution apparente de l'effectif due aux migrations lors de la fonte des neiges; cette donnée doit être mise à l'écart pour l'évaluation de la production.

- La valeur de l'ordonnée à l'origine peut être obtenue en prolongeant le tracé des courbes, c'est-à-dire en appliquant aux larvules récemment écloses le même taux de diminution des nombres qu'aux larves plus âgées. Cette extrapolation donne un nombre initial d'individus de 1320 en 1971 ef de 1350 en 1972. La production est alors respectivement de $62,2 \mathrm{mg} / \mathrm{m}^{2}$ et de $74 \mathrm{mg} / \mathrm{m}^{2}$; les rapports $\mathrm{P} / \mathrm{B}$ max. étant de 1,4 et 1,14 . Nous considèrerons ces valeurs comme les valeurs minimales de production vraisemblables.

La valeur de l'ordonnée à l'origine peut aussi être déduite du nombre théorique d'œufs pondus ( 155 en moyenne par femelle). Le nombre d'émergences au $\mathrm{m}^{2}$ fut de 148 en 1971 , de 113 en 1972 avec un sex-ratio égal à 1. Par contre, les femelles ne représentaient que $35 \%$ de la population capturée par chasse. Ceci suggère une migration (d'un tiers environ) des femelles, phénomène fréquent en montagne (Thomas 1975). Cette hypothèse admise, le nombre théorique d'œufs déposés par $\mathrm{m}^{2}$ de ruisseau est de 8030 en 1971 et 6130 en 1972 . La production serait alors de 84,6 en 1971 , de 90,8 en 1972 ; les rapports $\mathrm{P} / \mathrm{B}$ max. étant respectivement de 1,9 et 1,4 . Ces valeurs doivent être considérées comme les valeurs maximales de production.

L'étude successive des différentes cohortes amène aux conclusions suivantes :

1) la production varie peu d'une année à l'autre, les différences enregistrées ne dépassent pas $20 \%$ de la valeur maximum ;

2) la majeure partie de la production s'effectue lors de la deuxième année de développement, 80 à $60 \%$ selon l'hypothèse de départ. Le rapport Production $1^{\text {re }}$ année/Production $2^{\circ}$ année semble assez constant pour être appliqué aux cohortes 1970 et 1973 qui n'ont été suivies qu'une année seulement. Les valeurs obtenues sont très comparables aux résultats précédents ;

3) les rapports $P / B$ max. sont du même ordre que ceux trouvés jusqu'ici dans les Pyrénées centrales. 


\section{3. - DISCUSSION}

Le cycle de Capnioneura brachyptera est essentiellement caractérisé par :

1) une période de repos à l'état d'œuf ou de larves très jeunes ;

2) un taux de croissance très faible des jeunes larves après l'éclosion ;

3) une stagnation hivernale de la croissance suivie d'une reprise rapide au second printemps.

Les deux premiers points peuvent être mis en parallèle avec les stades de repos rencontrés chez les Capniidés (Khoo 1964, Harper 1973 ) en particulier dans le genre Capnioneura ( $C$. mitis espèce de plaine, Harper comm. verb., $C$. petitpierrae, espèce tunisienne, Berthélémy 1973). Effectivement il existe chez Capnioneura brachyptera un stade de repos, sans doute à l'état d'ouf, peut-être de jeunes larves non capturées par suite de la nature rocheuse du lit - Coleman et Hynes (1970) ont prouvé l'enfoncement de la faune lors de cette phase —. Si une telle diapause larvaire a lieu, elle permettrait d'expliquer la faiblesse de la croissance et la pauvreté de l'effectif jeune de la population. Le déterminisme de la période de repos, quelle qu'elle soit, reste toutefois difficilement explicable. Chez les Capniidés, en effet, la diapause permet aux larves de supporter les fortes températures estivales; d'autre part le cycle est généralement annuel : il n'existe donc pas de génération âgée durant l'été. La particularité de Capnioneura brachyptera réside essentiellement dans la différence du taux de croissance qui affecte les larves jeunes et âgées soumises aux mêmes conditions écologiques générales. Le régime alimentaire n’intervient pas : il reste identique (absorption de détritus et débris végétaux) durant le développement.

L'absence de développement hivernal suivie d'une reprise printanière rapide de la croissance est rare chez les Capniidés pour lesquels l'hiver constitue généralement une période de développement privilégié. Cette évolution qui a toutefois été observée par Svensson (1966) sur Capnia atra constitue un phénomène fréquent chez les espèces montagnardes.

L'évolution de la population en début de cycle biologique est difficile à préciser, nos prélèvements s'étant révélés défectueux dans la capture des premiers stades larvaires.

Selon l'estimation théorique de la densité larvaire initiale (à partir du nombre d'œufs pondus ou d'après le taux de diminution des nombres) la mortalité peut être à cette époque très forte ou relativement faible. De toutes façons, les observations basées directement sur les nombres obtenus par la suite montrent que l'effectif d'une cohorte décroît peu jusqu'à l'émergence (donc durant 
plus d'une année). Cette faible mortalité n'est peut-être qu'apparente si les premières populations larvaires récoltées sont sousestimées à la suite des migrations envisagées plus haut. Si elle est réelle, elle est favorisée : 1) par la configuration du ruisseau qui détermine à la station un apport par dérive certainement supérieur aux pertes ; 2) par le nombre réduit de prédateurs représentés uniquement par quelques stades jeunes d'Isoperla viridinervis et de Rhyacophila spp.

Les rapports $\mathrm{P} / \mathrm{B}$ max. sont relativement constants pour les cohortes successives. Que l'on considère les plus fortes ou les plus faibles estimations de production, les rapports $P / B$ max. sont voisins de ceux trouvés par Laville 1971 el 1975, Giani et Laville 1973, Décamps et Lafont 1974 sur des insectes aquatiques holométaboles de la même région. L'obtention d'une valeur similaire chez un insecte hétérométabole permet donc d'envisager l'utilisation d'un rapport $\mathrm{P} / \mathrm{B}$ max. compris entre 1 et 2 pour le calcul de la production des insectes aquatiques des Pyrénées centrales.

\section{TRAVAUX CITÉS}

BERTHÉLÉMY (C.). 1966. - Recherches écologiques et biogéographiques sur les Plécoptères et Coléoptères d'eau courante (Hydraena et Elminthidae) des Pyrénées. Annls limnol., 2 (2) : 227-458.

Berthét.́my (C.). 1969. - Les Capnioneura des Pyrénées. Annls limnol., 5 (1) : 25-47.

Berthélémy (C.). 1973. - Données préliminaires sur les Plécoptères de Tunisie. Verh. internat. Verein. Limnol., 18 : 1544-1548.

Coleman (M. J.), Hynes (H. B. N.). 1970. - The life histories of some Plecoptera and Ephemeroptera in a Southern Ontario stream. Canad. J. Zool., $48:$ 1333-1339.

Décamps (H.) et LAFont (M.). 1974. - Cycles vitaux et production des Micrasema pyrénéennes dans les mousses d'eau courante (Trichoptera, Brachycentridac). Annls Limnol., 10 (1) : 1-32.

Elliot (J. M.). 1973. - The life cycle and production of the leech Erpobdella octoculata (L.) (Hirudinea : Erpobdellidae) in a lake district stream. J. Anim. Ecol., 42 : 435-448.

Giani (N.) et Laville (H.). 1973. - Cycle biologique et production de Sialis lutaria L. (Mégaloptère) dans le lac de Port-Bielh (Pyrénées centrales). Annls limnol., 9 (1) : 45-61.

Harper (P. P.). 1972. - Life-histories of Capniidae and Taeniopterygidae (Plecoptera) in Southern Ontario. Arch. Hydrobiol., suppl. 40 (3) : 274-314.

KAJAK (Z.). 1967. - Remarques sur les méthodes d'investigations de la production du benthos (polonais avec résumé anglais). Ekol. Pol., sér. B, XIII (2) : 173-195.

Kноo (S. G.). 1964. - Studies on the biology of Capnia bifrons (Newman) and notes on the diapause in the nymphs of this species. Gewäss. Abwäss., 34/5 : 23-30. 
Lavandier (P.). 1974. - Écologie d'un torrent pyrénéen de haute montagne. I. Caractéristiques physiques. Annls limnol., 10 (2) : 173219.

Laville (H.). 1971. - Recherches sur les Chironomides (Diptera) lacustres du massif de Néouvielle (Hautes-Pyrénées). Deuxième partie : Communauté et Production. Annls limnol., 7 (3) : 335-414.

Laville (H.). 1975. - Production d'un Chironomide semivoltin (Chironomus commutatus Str.) dans le lac de Port-Bielh (Pyrénées centrales). Annls limnol., 11 (1) : 65-77.

Svensson (P. O.). 1966. - Growth of nymphs of stream living stoneflies (Plecoptera) in northern Sweden. Oikos, 17 : 197-206.

Thomas (A. G. B.). 1975. - Éphéméroptères du Sud-Ouest de la France. I. Migrations d'imagos à haute altitude. Annls limnol., 11 (1) : 47-66. 